\title{
Diversidad y ecología de macrohongos en la Reserva Llancahue: un sitio prioritario para la conservación
}

\author{
(Diversity and ecology of macrofungi in the Llancahue Reserve: \\ a critical place for conservation)
}

\author{
D. Barría-Díaz ${ }^{1 *}$, V. Salazar-Vidal ${ }^{2} \&$ E. Valenzuela ${ }^{3} \dagger$ \\ ${ }^{1}$ Instituto de Ciencias de la Educación, Facultad de Filosofía y Humanidades, \\ Universidad Austral de Chile. Independencia 631, \\ Valdivia, Región de los Ríos, Chile. \\ ${ }^{2}$ Laboratorio de Química de Productos Naturales, Facultad de Ciencias \\ Naturales y Oceanográficas, Universidad de Concepción. \\ Víctor Lamas $N^{\circ} 1290$, Concepción, Chile. \\ ${ }^{3}$ Instituto de Bioquímica y Microbiología, Facultad de Ciencias. \\ Universidad Austral de Chile. Independencia 631, \\ Valdivia, Región de los Ríos, Chile. \\ *Autor para correspondencia: daniela.barria@uach.cl \\ RECIBIDO: 16 de Mayo de 2019 \\ APROBADO: 31 de Mayo de 2019
}

DOI: $10.22370 /$ bolmicol.2019.34.1.1741

LOS AUTORES DECLARAN NO TENER CONFLICTO DE INTERESES

Palabras claves: hongos comestibles; biodiversidad; conservación.

Key words: edible fungi; biodiversity; conservation.

\section{RESUMEN}

Llancahue corresponde a un fragmento de bosque templado lluvioso, que se constituye en una verdadera reserva de la biodiversidad ubicada en la zona costera de la Región de Los Ríos. Con una superficie aproximada de 1.300 hectáreas, no sólo constituye la principal fuente de agua para la ciudad de Valdivia, sino que también presenta uno de los bosques más antiguos de la Provincia, por lo cual la Reserva Llancahue fue declarada recientemente como área protegida con fines de conser- vación y desarrollo sostenible. Debido a la escasa información existente sobre la diversidad de hongos presentes en la Reserva, se realizó muestreos estacionales durante el año 2016 e inicios del año 2017, con el fin de realizar un levantamiento de información sobre el componente hongos en este ecosistema boscoso. Los resultados obtenidos corresponden a un total de 38 especies de hongos determinadas de las cuales 23 son saprótrofas $(50 \%)$, 11 ectomicorrícicas (28\%) y 4 parásitas (13\%), siendo otoño la época con mayor fructificación de hongos. 


\begin{abstract}
Llancahue corresponds to a fragment of temperate rain forest, which constitutes a true reserve of biodiversity located in the coastal zone of the Los Ríos Region. With an approximate area of 1,300 hectares, it not only constitutes the main source of water for the city of Valdivia, but also presents one of the oldest forests of the Province, for which the Llancahue Reserve was recently declared as a protected area for conservation and sustainable development. Due to the scarce existing information on the diversity of fungi present in the Reserve, seasonal sampling was carried out during 2016 and beginning of 2017, in order to conduct a survey of the fungal component in this forest ecosystem. The results obtained correspond to a total of 38 species of fungi determined of which 23 are saprotrophic (50\%), 11 ectomycorrhizic (28\%) and 4 parasitic (13\%), with autumn being the season with the highest fruiting of fungi.
\end{abstract}

\section{INTRODUCCION}

Los hongos son un grupo de organismos que han sido escasamente estudiados, se estima que a nivel mundial existen entre 2.2 a 3.8 millones, con 120.000 especies aceptadas (Hawksworth \& Lücking, 2017). Los hongos juegan roles clave en el ciclo de carbono y el reciclaje de nutrientes, así como también, en los ecosistemas terrestres donde se comportan como especies mutualistas (micorrizas y líquenes), parásitas y saprótrofas (McLaughlin \& Spatafora, 2014). Cassman et al., (2016) sugieren que la biota fúngica desempeña un papel fundamental en la rizósfera, donde interviene en muchos procesos que influyen en el crecimiento de las plantas y la salud del suelo.

En los últimos años se ha incrementado el interés por estudiar los hongos presentes en nuestro país, donde se han registrado y descrito alrede- dor de 3.000 especies (Mujica \& Vergara, 1980; Moser \& Horak, 1975; Garrido, 1986; 1988); sin embargo, la información disponible respecto a su diversidad no está actualizada y es insuficiente como para establecer un número exacto del total de especies existentes en nuestro país. Recordemos que el micelio de los hongos está presente en los bosques durante todo el año; sin embargo, sus cuerpos fructíferos se observan principalmente en otoño e invierno, donde las condiciones ambientales como la alta humedad, precipitaciones abundantes y temperatura permiten su fructificación (Talley et al., 2002). El clima de Llancahue está clasificado como templado-lluvioso, presentando una cantidad significativa de precipitaciones a lo largo del año (Luebert \& Pliscoff, 2018), teniendo una temperatura promedio de $11.3^{\circ} \mathrm{C}$ y una media de precipitaciones de $2.179 \mathrm{~mm}$ (Moorman et al., 2013).

El conocimiento de las especies presentes en una zona geográfica determinada es una prioridad para mantener actualizadas las bases de datos sobre biodiversidad y atender la creciente demanda de información sobre los ecosistemas (Meyer et al., 2015). Por otra parte, la utilización de recursos bióticos hace necesario tener listas de las especies registradas hasta la fecha, sus usos potenciales y el grado de amenaza que representan (Botrill et al., 2011; Maron et al., 2015). Recientemente, se han realizado estudios sobre comunidades animales y vegetales presentes en la Reserva Llancahue que han aportado datos interesantes sobre la diversidad y la biogeografía de las especies (Donoso et al., 2014), no obstante, aún no se conocen los hongos presentes en esta zona geográfica y su relación con la vegetación predominante. Además, no existe información sobre la micosociología de este bosque, ni sobre la recolección de hongos comestibles por parte de las comunidades aledañas y cómo esta actividad se ve afectada por los incendios, tala de árboles, entre otros factores. 


\section{MATERIALES Y MÉTODOS}

\section{Descripción del área de estudio y vegetación predominante}

La Reserva Llancahue se localiza entre los $39^{\circ} 51^{\prime} 31.8^{\prime \prime}$ ' y $73^{\circ} 08^{\prime} 12.9^{\prime \prime} O$ a una altitud de 323 msnm, a $6 \mathrm{~km}$ al sureste de la ciudad de Valdivia y ocupa una superficie aproximada de 1.300 ha rodeada de plantaciones forestales (Fig. 1), siendo un área importante para la conservación, ya que provee de agua potable a la ciudad de Valdivia y los bosques que allí se desarrollan corresponden a uno de los más antiguos de la Provincia de Valdivia (Moorman et al., 2013). Está conformada por un bosque nativo adulto de tipo forestal siempreverde, donde las especies más representativas son Nothofagus dombeyi Mirb. Oerst. y Drimys win- teri (J.R. Forst y G. Forst). Además, la Reserva Llancahue posee otros dos tipos de bosque adulto, uno conformado por las especies Eucryphia cordifolia Cav., Laureliopsis philipiana (Looser) R. Schodde) y Aextoxicom punctatum (Ruiz \& Pav) y otro compuesto por árboles renovales de $N$. dombeyi (Donoso et al., 2014; Luebert \& Pliscoff, 2018). Dentro de la cuenca de Llancahue hay un fragmento nativo de bosque templado (cerca de 700 ha), que representa parte de un "hotspot" de biodiversidad y un ecosistema amenazado reconocido internacionalmente (Myers et al., 2000; Moorman et al., 2013). Estos bosques están formados principalmente por Ulmo (Eucryphia cordifolia), Tepa (Laureliopsis philippiana, Olivillo (Aextoxicon punctatum) y Canelo (Drimys winteri) y en ocasiones, Coihue (Nothofagus dombeyi) en posiciones emergentes.
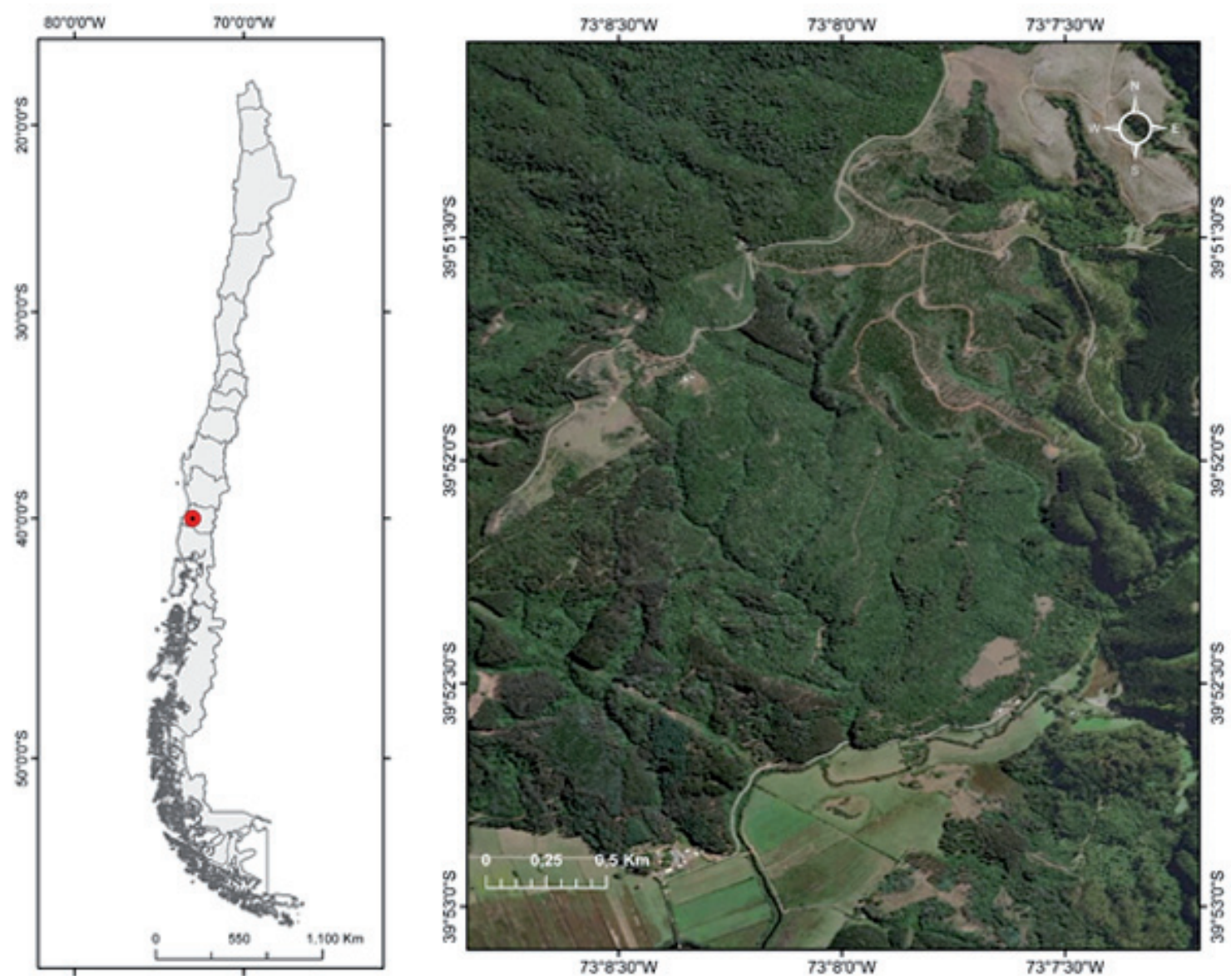

Figura 1. Mapa de la Reserva Llancahue donde se observa la vegetación predominante. 


\section{Recolección de material biológico}

La recolección de los ejemplares considerados en este estudio se realizó a través de muestreos estacionales durante el año 2016 e inicios del año 2017 en la Reserva Llancahue, Región de Los Ríos, donde se efectuó un levantamiento de información sobre la diversidad de especies de macrohongos presentes en este ecosistema boscoso. Para cada ejemplar recolectado en terreno se registró: fecha, localidad, coordenadas, sustrato, vegetación predominante y caracteres macroscópicos relevantes, tales como; aroma, textura del píleo, consistencia del estípite, tamaño, forma, color, presencia o ausencia de anillo, de volva, etc. Adicionalmente, se tomaron fotografías in situ de cada ejemplar con una EOS Rebel T6 EF y se colocó cada muestra en una bolsa de papel o papel alusa foil con su respectiva etiqueta. Se recolectaron ejemplares completos, evitando aquellos en proceso de descomposición o deteriorados debido a las condiciones climáticas. Finalmente, las muestras se trasladaron al Instituto de Bioquímica y Microbiología de la Universidad Austral de Chile para su posterior determinación microscópica.

\section{Procesamiento e identificación de las muestras}

Las muestras fueron deshidratadas a no más de $45^{\circ} \mathrm{C}$ utilizando un deshidratador de alimentos marca Blanik para evitar su pudrición o contaminación. Posteriormente, se realizó la determinación microscópica que consiste en realizar preparaciones de cortes transversales y longitudinales de las diferentes partes del esporocarpo para observar bajo el microscopio, utilizando distintos reactivos (KOH, reactivo de Melzer, Floxina, Azul de Cresilo, etc.) que resalten las estructuras que caracterizan a las especies (tamaño, forma y color de las esporas, basidios, cistidios, setas e hifas, presencia de fíbulas) según los protocolos presentes en Singer (1986) y Wright \& Albertó (2002).

\section{RESULTADOS}

Se realizó un muestreo en distintas épocas del año 2016 y uno a principios del año 2017, siendo otoño la estación con mayor presencia y abundancia de fructificaciones. El total de especies de macrohongos determinadas corresponde a 38 , de las cuales 11 son ectomicorrícicas, 23 saprótrofas y 4 parásitas. Asimismo, se destaca la presencia de 15 especies comestibles (Tabla 1 ).

Las especies ectomicorrícicas (11 especies) corresponden a un $28 \%$ total de las especies registradas para el ecosistema boscoso de la Reserva Llancahue (Ver Fig. 2), las cuales están representadas por los géneros: Cortinarius, Paxillus, Boletus, Ramaria, Amanita y Russula; se registró dos especies del género Cortinarius; sin embargo, existe potencial de recolectar más especies de este género realizando muestreos más frecuentes en la zona. La presencia de especies de Cortinarius, indica que existe una estrecha relación entre hongos de este género y especies de Nothofagus, como lo menciona Moser \& Horak (1975) quienes advirtieron para Chile y Argentina, la asociación obligatoria de las especies del género Cortinarius a estos árboles. Además, de acuerdo al estudio realizado por Valenzuela et al., (1998) en la Cordillera Pelada ubicada en la XIV Región de Chile, el género Cortinarius presenta una mayor cantidad de especies asociadas a $\mathrm{No}$ thofagus dombeyi y Nothofagus obliqua. Además, considerando lo expuesto por Salazar \& Palfner (2015), se deduce que los bosques de Nothofagus siempreverde suelen ser los hábitats preferidos para las especies del género Cortinarius que habitan en Chile, donde sus fructificaciones son muy abundantes.

Otro género micorrícico que se registró fue Amanita, destacando la abundancia de Amanita aurantiovelata y Amanita rubescens. Para el caso Amanita aurantiovelata, Valenzuela et al., (1998) 
Diversidad y ecología de macrohongos en la Reserva Llancahue: - Barría-Díaz D. et al

Tabla 1. Diversidad, procedencia, rol ecológico y estación de recolección de las especies de macrohongos recolectadas en la Reserva Llancahue durante el año 2016 y 2017. En amarillo se destaca la presencia de los hongos silvestres comestibles presentes en la Reserva.

\begin{tabular}{|c|c|c|c|}
\hline Especie & Procedencia/Distribución & Rol Ecológico & Estación de recolección \\
\hline Aleuria aurantia & Cosmopolita & Saprófito & otoño \\
\hline Aleurodiscus vitellinus & Nativo & Parásito & otoño \\
\hline Amanita aurantiovelata & Nativo & Ectomicorriza & verano \\
\hline Amanita diemii & Nativo & Ectomicorriza & invierno, verano \\
\hline Amanita rubescens & Introducido & Ectomicorriza & verano \\
\hline Anthracophyllum discolor & Nativo & Saprófito & otoño \\
\hline Austropaxillus sp. & Nativo & Ectomicorriza & invierno \\
\hline Boletus loyita & Endémico & Ectomicorriza & otoño, verano \\
\hline Butyriboletus loyo & Endémico & Ectomicorriza & otoño, verano \\
\hline Calocera cornea & Cosmopolita & Saprófito & otoño \\
\hline Chlorociboria aeruginascens & Cosmopolita & Saprófito & otoño \\
\hline Chlorophyllum rhacodes & Cosmopolita & Saprófito & otoño \\
\hline Clitocybe nuda & Cosmopolita & Saprófito & invierno \\
\hline Coprinopsis atramentaria & Cosmopolita & Saprófito & invierno \\
\hline Cortinarius sp. & Desconocida & Ectomicorriza & otoño, invierno \\
\hline Cortinarius magellanicus & Nativo & Ectomicorriza & otoño \\
\hline Cyttaria darwinii & Nativo & Parásito & otoño, primavera \\
\hline Favolaschia sp. & Desconocida & Saprófito & otoño \\
\hline Fistulina antarctica & Nativo & Parásito & invierno \\
\hline Ganoderma australe & Nativo & Parásito & invierno \\
\hline Guepiniopsis alpina & Nativo & Saprófito & otoño \\
\hline Hydropus dusenii & Nativo & Saprófito & invierno \\
\hline Hypholoma lateritium & Cosmopolita & Saprófito & invierno \\
\hline Marasmiellus alliiodorus & Nativo & Saprófito & otoño \\
\hline Marasmius sp. & Desconocida & Saprófito & otoño \\
\hline Mycena cyanocephala & Nativo & Saprófito & otoño \\
\hline Mycena galericulata & Cosmopolita & Saprófito & otoño \\
\hline Mycena pura & Cosmopolita & Saprófito & otoño \\
\hline Neoclitocybe byssiseda & Cosmopolita & Saprófito & otoño \\
\hline Plectania chilensis & Endémico & Saprófito & verano \\
\hline Pleurotus ostreatus & Cosmopolita & Saprófito & otoño \\
\hline Psilocybe aurantiaca & Desconocida & Saprófito & otoño \\
\hline
\end{tabular}


Diversidad y ecología de macrohongos en la Reserva Llancahue: - Barría-Díaz D. et al

\begin{tabular}{|c|c|c|c|}
\hline Ramaria flava & Cosmopolita & Ectomicorriza & otoño \\
\hline Russula nothofaginea & Nativo & Ectomicorriza & verano \\
\hline Russula sardonia & Introducido & Ectomicorriza & otoño \\
\hline Stereum sp. & Cosmopolita & Saprófito & primavera, verano \\
\hline Trametes versicolor & Cosmopolita & Saprófito & todo el año \\
\hline Tremella mesenterica & Cosmopolita & Saprófito & invierno \\
\hline
\end{tabular}

\section{Macrohongos presentes en la Reserva Llancahue de acuerdo a su rol ecológico}

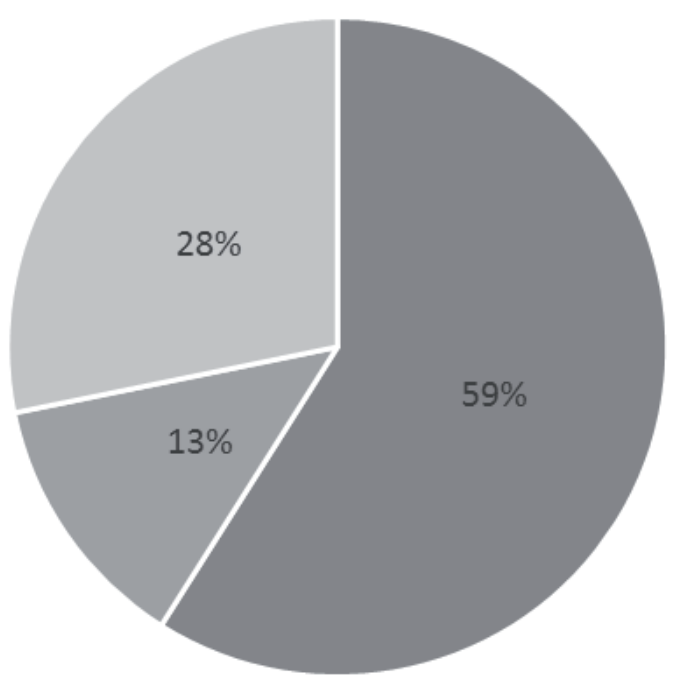

- Saprófito

- Parásito

Ectomicorriza

Figura 2. Representación de los porcentajes de especies de macrohongos presentes en la Reserva Llancahue de acuerdo a su rol ecológico.

señala que es una especie típica de los bosques de Nothofagus de la XIV Región ubicada en la depresión intermedia y con una distribución más restringida. En cambio, Amanita rubescens presenta una distribución geográfica amplia, cosmopolita y descrita para la Región de Los Lagos y de Los Ríos, la cual se asocia a los géneros Abies, Picea, Pinus spp., Quercus y Fagus (Murakami, 1993; Neville \& Poumart, 2004, Niazi et al., 2009). En Chile, $A$. rubescens ha sido descrita asociada mayormente a especies exóticas como Pinus radiata y Quercus robur (Valenzuela et al., 1996), así como a Nothofagus (Valenzuela et al., 1998). Esta diversidad de hábitats que presenta esta especie puede estar relacionada a una baja especificidad para formar asociaciones micorrícicas, lo que le otorga un amplio rango para desplazar a algunas especies micorrícicas nativas como las del género Cortinarius en los bosques andinos (Romano et. al, 2017).

Esta observación que se señala anteriormente, se relaciona con lo registrado en los muestreos en la Reserva Llancahue, ya que se observó una gran abundancia de $A$. rubescens que se podría deber a la presión de las grandes extensiones de plantaciones exóticas (Pinus radiata) en los 
sectores aledaños a la Reserva, lo que se condice por Romano et al., (2017), quienes señalan que el crecimiento sostenido de la superficie utilizada para plantaciones forestales, favorece que $A$. rubescens pueda invadir los bosques de distintas especies de Nothofagus, lo que sería el punto de partida de la invasión de $A$. rubescens.

Por otra parte, respecto al porcentaje de las especies saprótrofas que corresponde a un 59\% de las especies determinadas correspondiendo a un total de 23 (Ver Fig. 2). Las especies registradas fueron: Mycena, Marasmius, Hypholoma, Neoclitocybe. La abundancia de especies saprótrofas asegura que en estos bosques existe una adecuada degradación de la materia orgánica vegetal, formación de humus y la recirculación de elementos químicos como el N, P, S, entre otros, a los ciclos biogeoquímicos correspondientes, como también, a la gran cantidad de restos vegetales (ramas, ganchos de árboles y troncos) que se depositan en el piso del bosque (Valenzuela et al., 1998).

Para el caso de especies parásitas no se registró especial abundancia correspondiendo a un 13\% (Ver Fig. 2), lo que en suma, da cuenta de que este ecosistema se encuentra en un buen estado de sanidad forestal. Las especies parásitas encontradas fueron: Cyttaria, Fistulina y Ganoderma. Asimismo, destacar que durante el verano de 2017 se registraron más lluvias que lo habitual en la Región de Los Ríos, por lo que se encontró algunos Myxomycetes, tales como: Stemonitis sp., Ceratiomyxa sp. y Fuligo septica. Estos organismos suelen fructificar inmediatamente luego de las lluvias, con una temperatura adecuada. Además, se registraron especies de hongos comestibles altamente recolectados, tales como: Butyriboletus loyo, Boletus loyita, Cyttaria espinosae y Ramaria flava, pero se desconoce si estas especies son recolectadas en la zona. De ser así, habría que promover técnicas de recolección sustentable para que este recurso no se agote.
Finalmente, la estación con mayor registro de especies resultó ser otoño, debido a la gran variación interanual que presenta la fructificación de los hongos, principalmente, por la influencia que ejercen diversos factores meteorológicos y ecológicos, siendo de gran importancia el inicio de las lluvias (Salazar, 2016).

En la Figura 3 se muestran las fotografías de algunas de las especies encontradas en la Reserva Llancahue, las cuales se destacan por sus distintas formas y colores.

\section{CONCLUSIONES}

El levantamiento de información respecto a la diversidad de especias de hongos presentes en los ecosistemas de Chile, cobra un valor sustancial para la generación de conocimiento y el aporte a nivel de política pública para la conservación de nuestros ecosistemas boscosos. Actualmente, el Ministerio de Medio Ambiente y colaboradores, lleva a cabo una iniciativa que busca realizar un catastro de especies de macrohongos y líquenes a nivel nacional, por cual estos registros permitirán tener información actualizada sobre las especies de hongos y líquenes presentes en Chile.

Por otra parte, el conocer las especies de hongos y sus relaciones ecosistémicas de un determinado territorio, nos permite asociar este conocimiento a nuestro contexto local ya sea a través de diversas prácticas como también incidir en el ámbito de la educación formal y no formal. De esta manera, se vincula la naturaleza y sociedad fomentando una perspectiva sistémica de relaciones. Hoy en día, es prioritario educarnos respecto a las especies que conforman nuestro patrimonio, de tal manera que podamos valorar nuestros ecosistemas y, asimismo, formar una ciudadanía crítica. Por este motivo y debido a la escasa información existente sobre la diversidad de hongos presentes en la Reserva Llancahue, se realizó muestreos es- 
Diversidad y ecología de macrohongos en la Reserva Llancahue: - Barría-Díaz D. et al
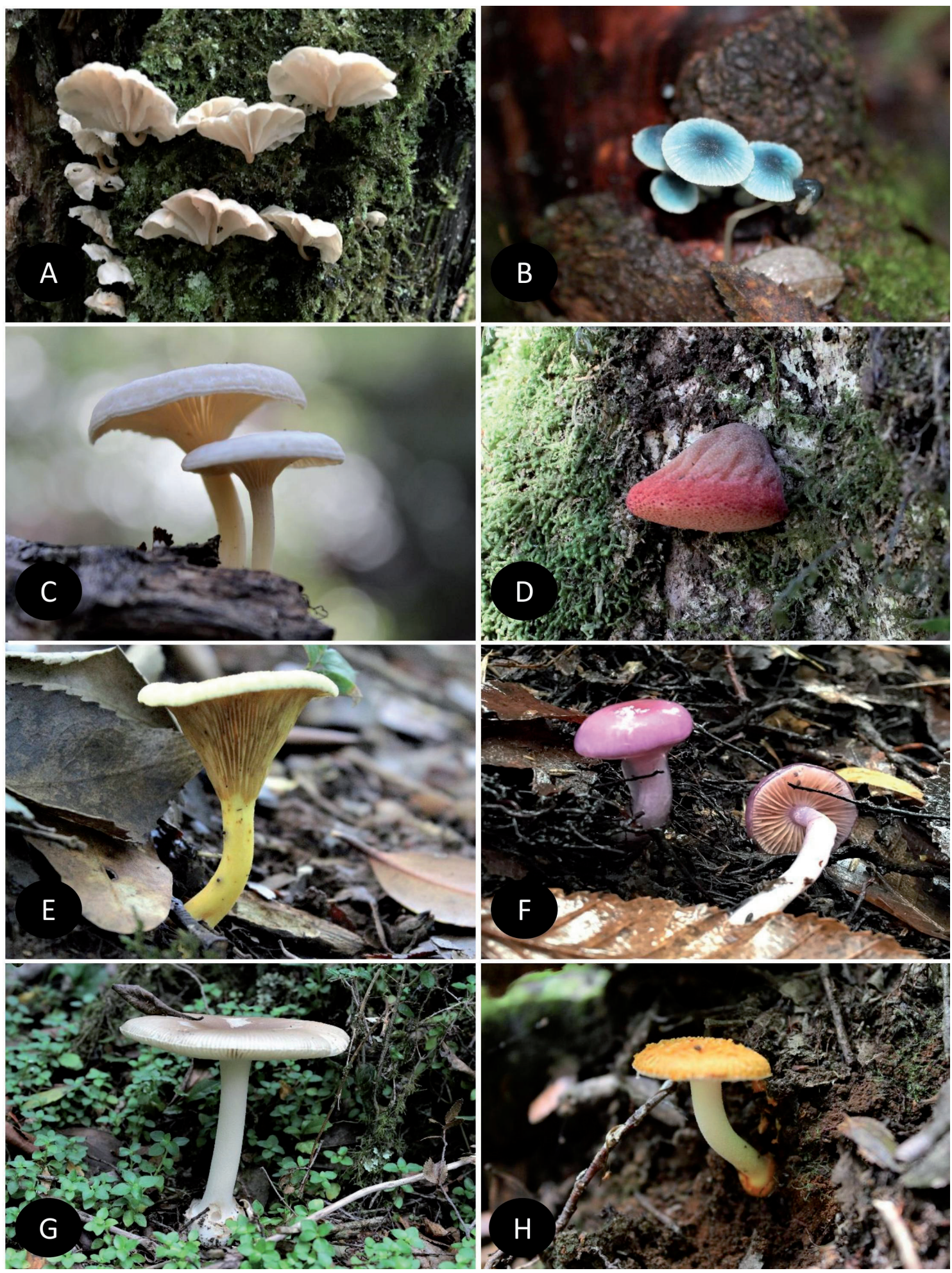

Figura 3. Algunas especies encontradas en la Reserva Llancahue. A) Marasmiellus alliiodorus, B) Mycena cyanocephala, C) Hydropus dusenii, D) Fistulina antarctica, E) Austropaxillus sp., F) Cortinarius magellanicus, G) Amanita diemii, H) Amanita aurantiovelata. 
tacionales durante el año 2016 e inicios del año 2017, que arrojaron como resultado un total de 38 especies de hongos determinadas, de las cuales 23 son saprófitas $(50 \%), 11$ ectomicorrícicas (28\%) y 4 parásitas (13\%). Cabe señalar que se presentó una mayor abundancia de especies en la estación de otoño, lo cual coincide con la literatura científica respecto al aumento de fructificaciones para esta estación.

Las ectomicorrizas más representativas en la Reserva Llancahue corresponden a los géneros de Cortinarius, Paxillus, Boletus, Ramaria, Amanita y Russula, característicos de los ecosistemas boscosos de Nothofagus. Dentro de estas se destacan dos especies endémicas del género Boletus, que al ser comestibles son altamente recolectadas para consumo o comercialización, por lo cual tienen importancia social y económica. Asimismo, se encontró una especie introducida invasora, $A$. rubescens, que está adaptándose a bosques nativos compitiendo con hongos chilenos micorrícicos. Esto es preocupante para las especies de hongos nativos que además de verse amenazados por la fragmentación de sus hábitats, también se encuentran en peligro de perder su nicho por la invasión de especies asociadas a plantaciones forestales.
Finalmente, se puede mencionar que la Reserva Llancahue, además de proveer de agua a la ciudad de Valdivia y de ser uno de los ecosistemas boscosos más antiguos de la Provincia de Valdivia, guarda una información relevante respecto a las diversas relaciones ecosistémicas que se establecen en este territorio, como es el caso de los hongos. De esta manera, es urgente y necesario llevar a cabo investigaciones científicas en este sitio, no sólo para aportar a la generación de conocimiento taxonómico-ecológico, sino también para potenciar esta Reserva realizando actividades en torno a la educación formal y no formal.

\section{AGRADECIMIENTOS}

Se agradece el aporte realizado por el Dr. Eduardo Valenzuela (Q.E.P.D), profesor titular del Instituto de Bioquímica y Microbiología, Facultad de Ciencias, Universidad Austral de Chile, quien formó parte importante en la orientación de este artículo, brindando su ayuda en la determinación de algunas especies. A MSc. María José Dibán de la Universidad de Chile, por la elaboración del mapa que incluye la ubicación geográfica y la vegetación predominante presente en la Reserva Llancahue. A la ONG Micófilos por la colaboración de sus profesionales en la revisión del artículo.

\section{REFERENCIAS}

Bottrill, M. C., Hockings, M. \& H. Possingham. 2011. In pursuit of knowledge: addressing barriers to effective conservation evaluation. Ecology and Society 16(2): 14 .

Cassman, N., Leite, M., Pan, Y., Hollander, M., Vvan Veen, J. \& K. Kuramae. 2016. Plant and soil fungal but not soil bacterial communities are linked in long-term fertilized grassland. Scientific Reports 6: 23680.

Donoso, P., Frene, C., Flores, M., Moorman, M., Oyarzun, C. \& J. Zavaleta. 2014. Balan- cing wáter supply and old-growth forest conservation in the lowlands of south-central Chile through adaptive co-management. Landscape Ecol. 29:245-260.

Garrido, N. 1986. Survey of ectomycorrhizal fungi associated with exotic forest trees in Chile. Nova Hedwigia 43: 423-442.

Garrido, N. 1988. Agaricales und ihre mykorrhizen in den Nothofagus-Wáldem mittelchiles. Bibliotheca Mycologica tomo 120, J. Cramer, Berlín, Stuttgart, Alemania, 528 pp. 
Hawksworth, D. \& R. Lücking. 2017. Fungal diversity revisited: 2.2 to 3.8 million species Microbiology Spectrum, 5 .

Luebert, F. \& P. Pliscoff. 2018. Sinopsis bioclimática y vegetacional de Chile. 2da. Edición. Santiago de Chile: Editorial Universitaria. 381 pp.

Maron, M., Gordon, A., Mackey, B., Possingham, H. \& J. Watson. 2015. Stop misuse of biodiversity offsets. Nature, 523, 401-403.

McLaughlin, D. \& J. Spatafora (eds). 2014. The Mycota 7. Systematics and Evolution part A. Springer, Heidelberg.

Meyer, C., Kreft, H., Guralnick, R. \& W. Jetz. 2015. Global priorities for an effective information basis of biodiversity distributions. Nature communications, 6,8221 .

Moorman, M., Donoso, P.J., Moore, S.E., Sink, S. \& D. Frederick. 2013 Sustainable protected area management: The case of Llancahue, a highly-valued periurban forest in Chile. Journal of Sustainable Forestry 32:783-805.

Moser, M. \& E. Horak. 1975. Cortinarius Fr. Und nahe verwandte Gattungen in Südamerika. Beih. Nova Hedwigia 52: 1-628.

Mujica, F. \& C. Vergara. 1980. Flora Fungosa Chilena. Segunda Edición. Ciencias Agrícolas, Facultad de Agronomía, Universidad de Chile, Chile. 308 pp.

Murakami, Y. 1993. Larger fungi from Northern Pakistan. In: T. Nakalke \& S. Malik (eds.). Cryptogamic Flora of Pakistan, Vol. 2: 105-147.

Myers, N., R.A. Mittermeier, C.G. Mittermeier, G.A.B. da Fonseca \& J. Kent. 2000. Biodiversity hotspots for conservation priorities. Nature 403:853- 858 .
Neville, P. \& S. Poumarat. 2004. Amaniteae. 1. Fungi Europaei 9: 1-1119.

Niazi, A. R., Iqbal, S. H., \& N. A. Khalid. 2009. Ectomycorrhizae between Amanita rubescens and Himalayan spruce (Picea smithiana) from Pakistan. Mycotaxon, 107(1), 73-80.

Romano G., Greslebin, A. \& B. Lechner. 2017. Hongos agaricoides de los bosques de Nothofagus pumilio (Chubut, Argentina): Clave y listado de especies. Rev. Mus. Argentino Cienc. Nat., n.s. 19(1): 39-69.

Romano, G. \& F. Kuhar. 2017. Dos registros de Agaricales (Basidiomycota) exóticos en bosques nativos de la Patagonia Argentina. Boletín Micológico 32(1): 9-12

Salazar, V. \& G. Palfner. 2015. Productividad del hongo nativo Cortinarius austroturmalis en bosques de Nothofagus siempreverde y caducifolio de las Reservas Nacionales Altos de Lircay y Los Ruiles de la VII Región, Chile. Boletín Micológico, 30(2): 28-39.

Salazar, V. 2016. Micosociología: Antecedentes históricos, evolución y proyecciones. Bol. Micol. 2016; 31(2): 23-35.

Singer, R. 1986. The Agaricales in Modern Taxonomy. 4th ed. Koeltz Scientific Books, Koenigstein. 981 pp.

Talley, S., Phyllis, D., \& T. Kursar. 2002. The effects of weather on fungal abundance and richness among 25 communities in the Intermountain West. BMC Ecology 2:7.

Valenzuela, E., Ramirez, C., Moreno, G., Polette, M., Garniga, S., Peredo, H., \& J. Grinbergs. 1996. Agaricales más comunes recolectados en el Campus Isla Teja de la Universidad Austral de Chile. Bosque, 17(1): 51-63. 
Valenzuela, E., Moreno, G., Garnica, S. \& C. Wright, J. \& E. Albertó. 2002. Hongos: Guía de Ramírez. 1998. Micosociología en bosques nativos la Región Pampeana. Edit. Colin Sharp. Buenos de Nothofagus y plantaciones de Pinus radiata en Aires. Argentina. 279 pp.

la X Región de Chile: diversidad y rol ecológico. Revista Chilena de Historia Natural. 71: 133 - 146. 